Journal of

Accident and

Emergency

Medicine 1994

12, $156-157$

\title{
Winging of the scapula: an unusual complication of chest tube placement
}

\author{
W.U.HASSAN ${ }^{1}$ and N.P. KEANEY ${ }^{2}$ \\ 'Leicester Royal Infirmary, Infirmary Square, Leicester and ${ }^{2}$ The Royal Infirmary, New Durham Road, \\ Sunderland
}

\section{SUMMARY}

Chest tube insertion is generally considered a safe procedure. We describe a patient who developed winging of the scapula following chest tube insertion. This complication has not been documented before.

Key words: chest tube placement, scapula winging

\section{CASE HISTORY}

A 24-year-old man was admitted to hospital from the accident and emergency (A\&E) department with a 1 month history of cough productive of green sputum, night sweats, anorexia, weight loss and generalized aches and pains. He smoked 20 cigarettes a day and had stopped smoking a few weeks before admission. He worked as a pipe fitter in the shipyards. His past medical history was unremarkable.

On examination he looked unwell and had a pyrexia of $37.5^{\circ} \mathrm{C}$. A chest radiograph showed consolidation of the right middle and lower lobes and a right pleural effusion. A diagnostic pleural tap revealed thick blood stained pus, which showed gram positive cocci on staining and grew Group $C$ Streptococcus haemoliticus on culture. Under local anaesthesia an incision was made in the mid-axillary line in the sixth intercostal space, and after blunt dissection a wide bore (size 30 ) chest tube was inserted.

He made a good recovery and the chest drain was removed 3 days later. On the fourth day, he complained of slight weakness in his right arm on abduction, but no abnormality was detected.

When reviewed in the clinic after 2 weeks, he had noticed a gradual worsening of the weakness in his right arm, particularly while lifting and pushing. There was no history of shoulder pain. On examination, there was no tenderness or atrophy of the shoulder girdle muscles. Winging of the right scapula was noted which was made worse when the patient was asked to push forward with the hands at the waist level. With regular physiotherapy, he gradually regained muscle strength and winging of the scapula disappeared after 6 months.

\section{DISCUSSION}

Previously described complications of chest drain insertion include: empyema, ${ }^{1}$ haemothorax, penetration through the diaphragm or spleen, delayed pulmonary perforation, ${ }^{2}$ unilateral pulmonary oedema, bronchopulmonary fistula, Horner's syndrome ${ }^{3}$ and phrenic nerve paralysis. ${ }^{4}$ Damage to the long thoracic nerve following chest tube insertion has not been described before.

Serratus anterior along with pectoralis minor, draws the scapula forwards and is the chief muscle concerned with all reaching and pushing movements. The long thoracic nerve $(C 5,6,7)$ innervates the serratus anterior segmentally. It lies behind the mid-axillary line on the surface of the muscle, deep to the fascia and segmentally innervates the serratus anterior, C5 into the upper two digitations, C6 into the next two and $\mathrm{C} 7$ into the lower four digitations (Fig. 1). Damage to the long thoracic nerve results in serratus anterior weakness, which gives the scapula a peculiar winged appearance. The patient is unable to raise the arm fully or to carry out pushing movements, and attempts to do so are followed by further projection of the lower end of the scapula. In this patient, damage to the long thoracic nerve possibly occurred due to the blunt dissection prior to the insertion of wide bore chest drain. When there is isolated damage to the $\mathrm{C} 7$ nerve root, as might be anticipated because of the chest tube insertion, winging may become more marked when pushing forward with the hands at the waist level. 
W.U. Hassan

\& N.P. Keaney

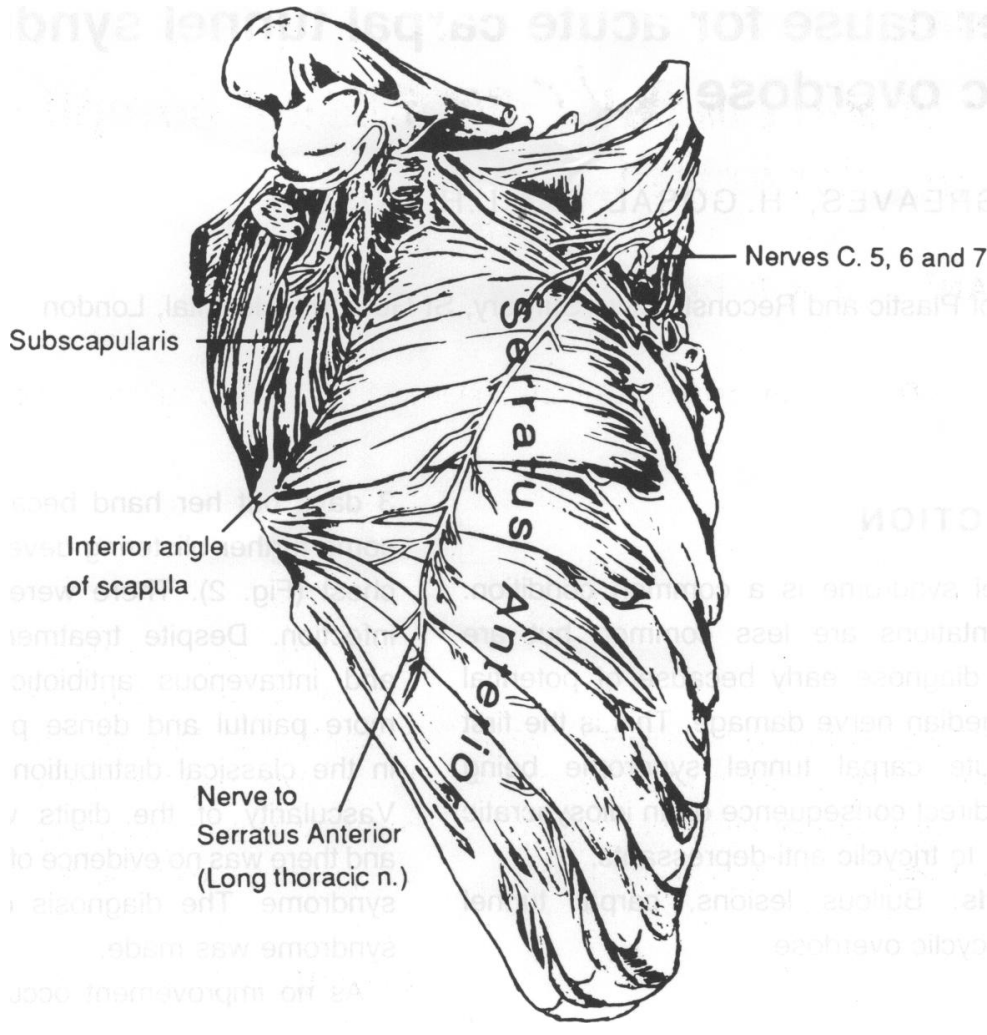

Fig. 1. Serratus anterior muscle with bony attachments and nerve supply.

\section{REFERENCES}

1. Millikan J.S., Moore E.E., Steiner E., Aragon G.E. \& Van Way III C.W. (1980) Complications of tube thoracostomy for acute trauma. American Journal of Surgery 140, 738-741.

2. Resnick D.K. (1993) Delayed pulmonary perforation: A rare complication of tube thoracotomy. Chest 103,
311-313

3. Bertino R.E., Wesby G.E. (1987) Johnson R. Horner's syndrome occurring as a complication of chest tube placement. Radiology 164, 745.

4. Oditta J.C., Khan A.S., Dincsoy M., Kayyali M., Masoud A. \& Ammari A. (1992) Neonatal phrenic nerve paralysis resulting from intercostal drainage of pneumothorax. Paediatric Radiology 22(5), 379-381. 